Jan Hendrik Carstens, Clemens Gühmann

\title{
Adaptive control of a boost-buck converter for thermoelectric generators
}

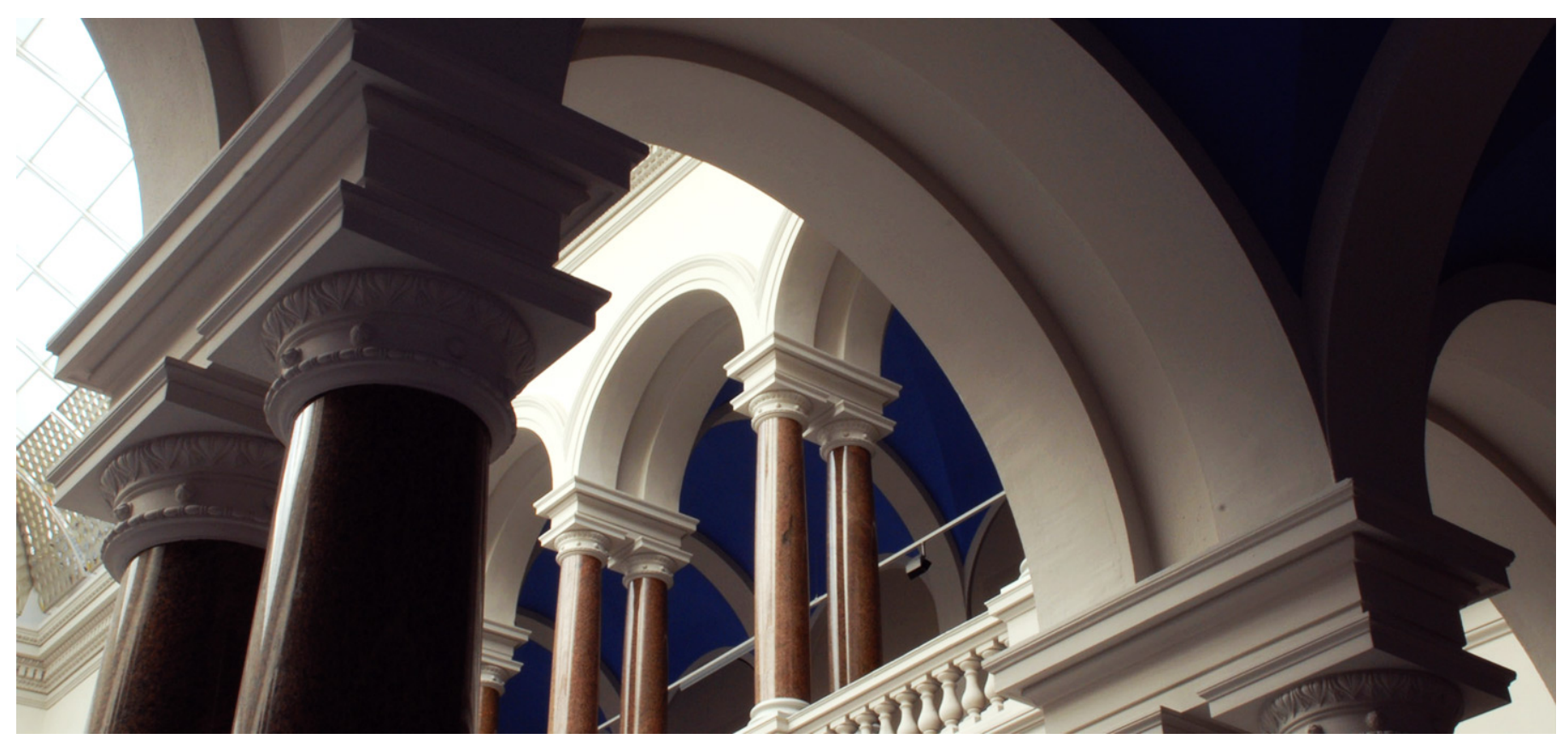

\section{Suggested Citation}

Carstens, Jan Hendrik; Gühmann, Clemens: Adaptive control of a boost-buck converter for thermoelectric generators. - In: European Control Association (Eds.): 2014 European Control Conference (ECC 2014) : Strasbourg, France, 24 - 27 June 2014. - Piscataway, NJ : IEEE, 2014. - ISBN: 978-3-9524269-1-3. - pp. 2121-2126. - DOI:10.1109/ECC.2014.6862328. (Postprint version is cited, page numbers differ.) 


\title{
Adaptive Control of a Boost-Buck Converter for Thermoelectric Generators
}

\author{
Jan Hendrik Carstens ${ }^{1}$ and Clemens Gühmann ${ }^{2}$
}

\begin{abstract}
Thermoelectric generators (TEGs) are used to recover waste heat of the exhaust gas and convert it into electric energy in automotive applications. The temperature of the waste heat influences the voltage and internal resistor of a TEG. For the electric linking of TEGs to the on-board power supply, a DC-DC converter may be used. The control of the DC-DC converter must be robust against dynamic changes and additionally has to track the maximum power point (MPP) of the TEG. This paper presents a digital cascade controller for a boost-buck converter to charge a vehicle battery and to supply the load. To track the MPP, a hill climbing (HC) algorithm is implemented, which is also used for photovoltaics. The conversion time of the $\mathrm{HC}$ is minimized with an adaptive step size. Width variations of electric parameters of TEG influence the dynamic and stability of the controllers. With a closed loop identification, the parameter variation is estimated, and the control parameters can be redesigned. An experimental result show the efficiency of the adaptive control.
\end{abstract}

\section{INTRODUCTION}

The growing mobility increases the world-wide fuel consumption. In contrast, the amount of fossil fuel is limited. Additionally, the environmental burden is increasing dramatically. Many governments have enacted laws to regulate and reduce the fuel consumption as well as the $\mathrm{CO}_{2}$ emission of combustion engines. Conventional combustion engines basically convert chemical energy stored in fossil fuel into mechanical energy. Unfortunately, during that process the main part of the energy (about 50\%) is disappearing as heat [1].

A recovery of this wasted thermal energy would create a high potential to save fuel and to reduce the environment burden. This unused exhaust gas wasted heat can be converted into electric power through a thermoelectric generator (TEG) [6], [7]. A TEG consists of several thermoelectric elements (TEs) with $\mathrm{n}$ - and p-type semiconductor materials [4], [15]. The thermoelectric power can be linked to the on-board power supply via DC-DC converters to charge the vehicle battery and supply the load. Consequently the efficiency of the combustion engine increases, because the alternator is relieved. To support different power classes of TEGs and on-board power supplies, a boost-buck converter is selected, with an input voltage range up to $60 \mathrm{~V}$ and a current range to $20 \mathrm{~A}$. The nominal power is $300 \mathrm{~W}$. A boost-buck converter

\footnotetext{
*This project "Thermoelektrische Generatoren 2020" (03X3553E) is supported from German ministry of education and research (BMBF).

${ }^{1}$ Jan Hendrik Carstens is research assistants of Electronic Measurement and Diagnostic Technology, Technische Universität Berlin, 10587 Berlin, Germany jan.h.carstensetu-berlin.de

${ }^{2}$ Clemens Gühmann is the head of Chair of Electronic Measurement and Diagnostic Technology, Technische Universität Berlin, 10587 Berlin, Germany clemens.guehmann@tu-berlin. de
}

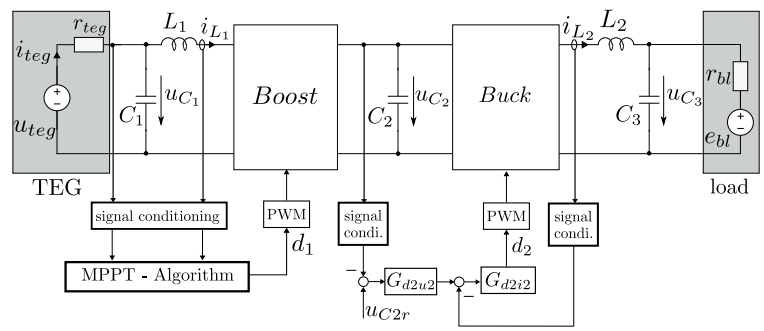

Fig. 1. Overview of control structure

has a general high efficiency, for a wide range of input power [1], [5]. Further, the output voltage of the converter is either greater than or less than the input voltage [1].

The converter has different requirements. Firstly, the TEG has to be operated in the maximum power point (MPP). A maximum power point tracking (MPPT) algorithm in general is based on a gradient descent and is used in particular for photovoltaic. This method can be applied to a TEG. An overview of different MPPT techniques, and their advantages and disadvantages, are presented by Esram [3]. Secondly, the power of the TEG is used to charge the battery and to support the power supply of a vehicle. The idea is to ensure a stable charging using a cascade control, which regulates the voltage of a storage capacity between boost and buck converter and charges the battery with a current control, see Fig. 1 [5]. Generally, the design of the control is based on the assumption that the internal resistor of a TEG is nearly constant and only the voltage depends on the temperature of the waste heat. However, the electric conductivity can be very different, depending on the materials. Additionally, the temperature at the TEG affects the internal resistor. An overview of the characteristic parameters of different materials is presented in [15], [14]. The internal resistor of a TEG limits the maximum current and influences the closed loop dynamic of the inner current loop of the cascade control. With a detailed knowledge of the characteristic of the TEG and especially the materials, it is possible to design control parameters, which are robust against parameter variations. However, the control of the boost-buck converter must be stable for each type of TEG. This paper proposes to use a recursive closed loop identification [8] with an autotuning controller to compensate the dynamic variations of the internal resistor of a TEG. In this context, this paper is organised as follows. The characteristic and model of the converter are analysed in Section 1. Section 2 briefly presents the digital control of the MPPT and the cascade control. In 


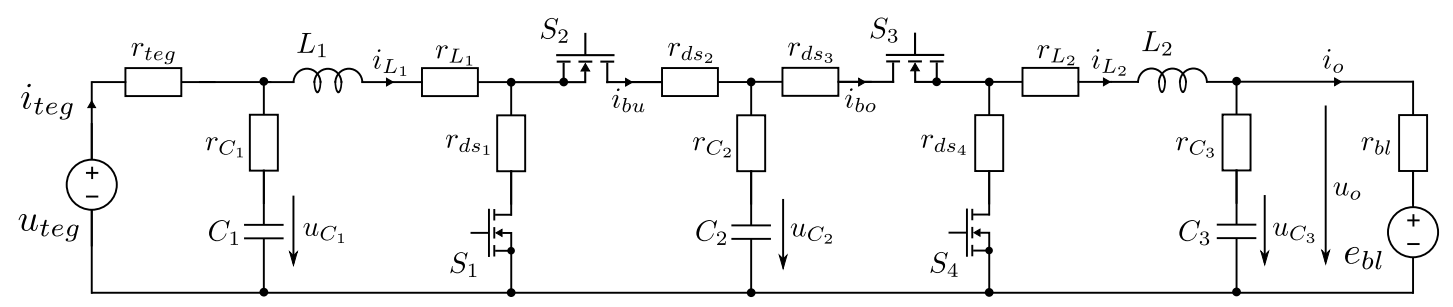

Fig. 2. Electric circuit of boost-buck converter with TEG and source.

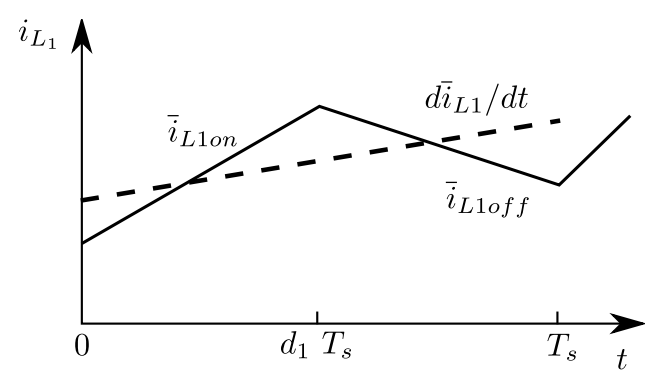

Fig. 3. Averaging of the inductor current.

Section 3, the recursive closed loop identification and the self-tuning algorithm is given. An experimental result are presented in Section 4. Finally, in Section 5 the results are discussed.

\section{Modeling Of Boost-Buck CONVERTER}

The physical principle of a DC-DC converter is based on charging and discharging of capacitors and inductors. The duration of charging and discharging is realised by using electric switching elements, like MOSFETs. As a result, the magnitude of current and voltage can be manipulated.

The boost-buck converter has four MOSFETs (see Fig. 2), whereas the switches $S_{1} / S_{2}$ and $S_{3} / S_{4}$ are synchronised. This means, if the switches $\mathrm{S} 1 / \mathrm{S} 3$ are closed, then $S_{2} / S_{4}$ are open. The MOSFETs are directly controlled by a PWM signal with a fixed frequency $f_{s}=1 / T_{s}$.

A switched mode converter is a nonlinear circuit, where different structures are periodically changed between on and off state of the MOSFETs. Fig. 3 shows the inductor current $i_{L 1}$. For duty cycle $d_{1}=t_{s 1 o n} / T_{s}$ the switch $S_{1}$ is turned on. In this interval the current $i_{\text {teg }}$ charges the inductor and the current $i_{L 1}$ increases with the averaged slope $\bar{i}_{L 10 n}$.

For the interval $d_{1}^{\prime}=t_{s 2 o n} / T_{s}=1-d_{1}$ the inductor current flows into the buck converter and the current $i_{L 1}$ decreases with $\bar{i}_{L 1 \text { off }}$. The states $\bar{i}_{L 1 o n}$ and $\bar{i}_{L 1 \text { off }}$ are weighted with duty cycle $d_{1}$ and $d_{1}^{\prime}$, thus results in the timeaveraged state:

$$
\frac{d \bar{i}_{L 1}}{d t}=d_{1} \frac{d \bar{i}_{L 1 o n}}{d t}+d_{1}^{\prime} \frac{d \bar{i}_{L 1 o f f}}{d t}
$$

This widely used approach is termed state-space-averaging (SSA), which are accurate for analyses up to $f_{s} / 2$ [13], [2]. It is assumed that the boost-buck converter can be separated into a boost converter with a current load $i_{b u}$ and

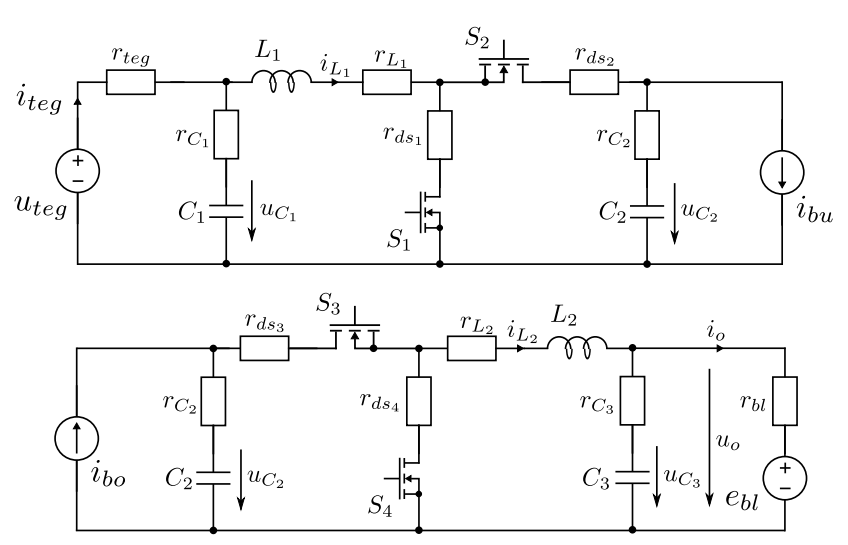

Fig. 4. Boost-buck converter separated in two converters with a constant current load/supply.

a buck converter with a current source $i_{b o}$ (see Fig. 4). This assumption is only valid for a nearly steady current.

With the replacement of $i_{b u}=d_{2} i_{L 2}$ and $i_{b o}=d_{1}^{\prime} i_{L 1}$, whereby $d_{1}$ describes the duty cycle of $S_{1}$ and $S_{2}$ and $d_{2}$ of $S_{3}$ and $S_{4}$, the state averaging of the converter is given by (1) - (5), with the parameters $\alpha, \beta, \gamma, \delta, \epsilon$ (see the Appendix).

$$
\begin{aligned}
\frac{d \bar{u}_{C 1}}{d t}= & \beta_{1} \bar{u}_{C 1}+\beta_{2} \bar{i}_{L 1}+\beta_{3} \bar{u}_{t e g} \\
\frac{d \bar{u}_{C 2}}{d t}= & \delta_{1} \bar{i}_{L 1}+\delta_{2} \bar{i}_{L 1} d_{1}+\delta_{3} \bar{i}_{L 2} d_{2} \\
\frac{d \bar{u}_{C 3}}{d t}= & \gamma_{1} \bar{i}_{L 2}+\gamma_{2} \bar{u}_{C 3}+\gamma_{3} \bar{e}_{B L} \\
\frac{d \bar{i}_{L 1}}{d t}= & \alpha_{1} \bar{i}_{L 1}+\alpha_{2} \bar{u}_{C 1}+\alpha_{3} \bar{u}_{C 2} \alpha_{4} \bar{u}_{t e g} \\
& +\alpha_{5} \bar{d}_{1} \bar{i}_{L 1}+\alpha_{6} \bar{d}_{2} \bar{i}_{L 2}+\alpha_{7} \bar{d}_{1} \bar{u}_{C 2} \\
& +\alpha_{8} \bar{d}_{1} \bar{d}_{2} \bar{i}_{L 2} \\
\frac{d \bar{i}_{L 2}}{d t}= & \epsilon_{1} \bar{i}_{L 2}+\epsilon_{2} \bar{u}_{C 3}+\epsilon_{3} \bar{e}_{B L}+\epsilon_{4} \bar{i}_{L 2} \bar{d}_{2} \\
& +\epsilon_{5} \bar{d}_{2} \bar{i}_{L 1}+\epsilon_{6} \bar{d}_{1} \bar{d}_{2} \bar{i}_{L 1}
\end{aligned}
$$

The averaged values are substituted by means of a DC and a small AC value [13]. The linearisation yields to the small signal state space model for the converter at the operation point.

$$
\dot{\boldsymbol{x}}=\boldsymbol{A x}+\boldsymbol{B u}
$$




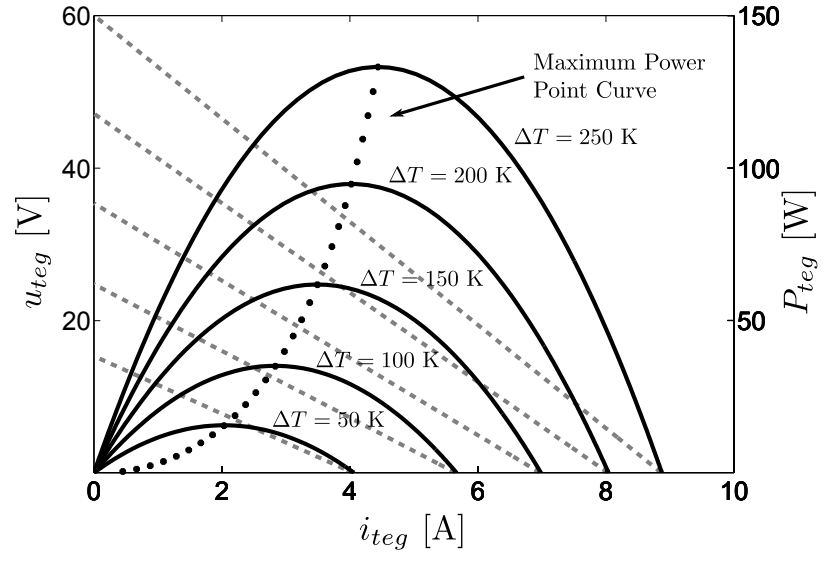

Fig. 5. Voltage and current characteristic of a simulated PbTe [4] TEG at different temperatures (gray dashed line) and the power curves (black line).

where $\boldsymbol{x}=\left[\tilde{u}_{C 1}, \tilde{i}_{L 1}, \tilde{u}_{C 2}, \tilde{i}_{L 2}, \tilde{u}_{C 3}\right]$ is the state vector and $\boldsymbol{u}=\left[\begin{array}{llll}\tilde{u}_{t e g}, & \tilde{e}_{B L}, & \tilde{d}_{1}, \tilde{d}_{2}\end{array}\right]$ is the input vector. For simplification the indices of the average values are neglected. Also, all measured signals are filtered with a second-order low pass. The cutoff frequency is $1 \mathrm{kHz}$. Based on this model, the system can be analysed for the design of the controller

\section{Boost-Buck CONVERTER CONTROL}

\section{A. Maximum Power Point Tracking of Boost Converter}

A TEG converts heat directly to electric energy. This effect is based on the Seebeck-Effect:

$$
u_{\text {teg }}=\left(\alpha_{n}-\alpha_{p}\right) \Delta T
$$

The Seebeck coefficients $\alpha_{n}$ and $\alpha_{p}$ are corresponding to the specific material of a $\mathrm{n}$ - and $\mathrm{p}$-doted semiconductor and $\Delta T$ is the temperature difference of the TEG [1]. Hence, changes of the temperature affect the voltage of the TEG, whereas the internal resistance $r_{\text {teg }}$ depends primarily on geometry and material [11]. The electric power of a TEG is limited by this internal resistance $r_{\text {teg }}$.

Fig. 5 shows the power curves from a TEG, for different temperature differences. It can be obtained, that the power has only one MPP, for each temperature. The MPPT finds automatically the current in which the TEG operates to obtain the maximum power and tracks this point at varying temperatures. One known and proven tracking algorithm is the $\mathrm{HC}$ [3]. The $\mathrm{HC}$ involves a perturbation of the duty cycle $d_{1}$, which influences the input current from the boost converter. The actual power $P(k)$ is calculated periodically and is compared to the previous power $P(k-1)$. For $\Delta P>0$ the duty cycle is decreased, and for $\Delta P<0$ it is increased. The MPP is obtained when $\Delta P=0$. It should be noticed, that the transfer function of $d_{1}$ to $i_{L 1}$ has a negative sign. The algorithm for the HC yields to

$$
d_{1}(k)=d_{1}(k-1)-\mu \Delta P
$$

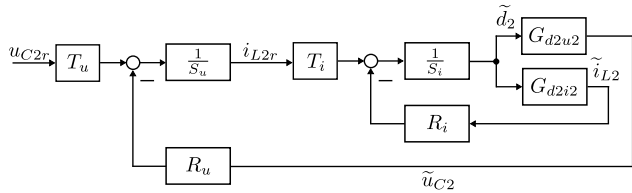

Fig. 6. Closed loop system of buck converter

where $\mu$ is the step size of the duty cycle. A disadvantage of the $\mathrm{HC}$ is the constant step size. The performance time for a small $\mu$ is long but for a large $\mu$ the HC can oscillate around the MPP. An adaptive step size algorithm compensates these effects:

$$
\mu(k)=\frac{|\Delta P|}{\mu(k-1)}
$$

The adaptive step size (9) will reduce $\mu$ for a large gradient $\Delta P$ and vice versa [17].

\section{B. Cascade Control of Buck Converter}

To charge the battery and supply the voltage of the load, a cascade control structure is used. By the control of the capacitor voltage $u_{C 2}$ between boost and buck converter it can be ensured, that fluctuations or changes of the TEG are compensated. The capacity $C_{2}$ functions as a buffer. A subsidiary control regulates the current $i_{L 2}$, which charge the vehicle battery. The advantages of a cascade control are the compensation of the disturbance by the fast current control and the higher settling time of the voltage controller to regulate the steady reference voltage $u_{C 2}$. A feature of this control structure is, that the reference voltage can be changed. This allows the use of the control structure for different battery voltage levels. [5]

From the discretisation of (1) - (6) the transfer function follows

$$
\begin{aligned}
G_{d 2 i 2}\left(q^{-1}\right) & =\frac{\tilde{i}_{L 2}}{\tilde{d}_{2}}=\frac{B_{i}\left(q^{-1}\right)}{A\left(q^{-1}\right)} \\
G_{d 2 u 2}\left(q^{-1}\right) & =\frac{\tilde{u}_{C 2}}{\tilde{d}_{2}}=\frac{B_{u}\left(q^{-1}\right)}{A\left(q^{-1}\right)}
\end{aligned}
$$

where $q^{-1}$ is the backward shift operator $\left(q^{-1} u(k)=u(k-\right.$ $1)$ ); $A, B_{i}$ and $B_{u}$ are the characteristic polynomials of fourth order. Fig. 6 shows the closed loop system, where $u_{C 2 r}$ is the reference voltage of the capacity $C_{2}$ and $i_{L 2 r}$ the desired output current.

The controller is based on a two-degrees-of-freedom digital controller [10], where the desired characteristic polynomial of the closed loop system is given by

$$
P_{c l}\left(q^{-1}\right)=A\left(q^{-1}\right) S\left(q^{-1}\right)+B\left(q^{-1}\right) R\left(q^{-1}\right)
$$

where $S$ and $R$ are filters. The filters can be divided in

$$
\begin{aligned}
S\left(q^{-1}\right) & =\bar{S}\left(q^{-1}\right) H_{s}\left(q^{-1}\right) I_{s}\left(q^{-1}\right) \\
R\left(q^{-1}\right) & =\bar{R}\left(q^{-1}\right) H_{r}\left(q^{-1}\right)
\end{aligned}
$$

where $I_{s}$ is an integrator to compensate the steady state error. $H_{s}$ and $H_{r}$ are pre-specified polynomials to compensate well damped zeros and poles. The result of the Diophantine 
equation of (12) gives $\bar{S}$ and $\bar{R}$. [10] With this design the current feedback control yields

$$
\begin{aligned}
H_{c l i} & =\frac{T_{i} B_{i}}{P_{c l i}} \\
P_{c l i} & =A S_{i}+B_{i} R_{i}
\end{aligned}
$$

where $H_{c l i}$ is the closed loop transfer function and $T_{i}$ is the DC gain compensation to receive $P_{c l i}(1)=1$. With (11) and (15), the transfer function from $\widetilde{i}_{L 2 r}$ to $\widetilde{u}_{C 2}$ yields to

$$
G_{i 2 u c 2}=\frac{T_{i} B_{u}}{P_{c l i} H_{s i}}=\frac{B_{i u}}{A_{i u}}
$$

where $H_{s i}$ is the pre-filter to cancel the well damped zeros of $G_{d 2 i 2}$. From (17) follows, that the desired characteristic of the current control influences the voltage dynamic $\widetilde{u}_{C 2}$. Especially the pre-filter $H_{s i}$ has an influence of the dynamic of $G_{i 2 u c 2} . H_{s i}$ is neglected, if the zeros of $G_{d 2 i 2}$ are not canceled or the canceled zeros are smaller than the dominant poles of $P_{c l i}$. The closed loop of the voltage control yields to

$$
\begin{aligned}
H_{c l u} & =\frac{T_{u} B_{i u}}{P_{c l u}} \\
P_{c l u} & =A_{i u} S_{u}+B_{i u} R_{u}
\end{aligned}
$$

where $T_{u}$ compensates the DC gain of the closed loop to one. The choice of the control parameters can further be specified by the output sensitivity function $S F\left(q^{-1}\right)$ and the complementary output sensitivity function $T F\left(q^{-1}\right)$ of the feedback-control [10]. Especially the sensitivity function $T F$ of the current controller is selected to damp high frequencies of the reference signals. The charging of the battery should be smooth and further, a fast changing of the current has a feedback on input current $i_{L 1}$ and the middle voltage $u_{C 2}$. A very quick change of the current amplitude could pull down the voltage $u_{C 2}$. As a result, the current $i_{L 1}$ flows directly via $S_{2}$ and $S_{3}$ into the battery.

\section{SElF-TUNing CONTROL OF THE CURRENT-LOOP}

The control for the boost-buck converter must be stable and the controller needs to be designed in such a way, that the stability is not affected by any variations of the internal resistor, the voltages on the TEG or hardware parameter. Fig. 7 shows the poles and zeros of the plants $G_{d 2 i 2}$ and $G_{d 2 u 2}$, whereas the characteristic polynomial $A$ is the same for both. The analysis of the poles and zeros makes clear that the system is stable for all values of the internal resistor. In the range of $0-0.4 \Omega$ the dominant pole of $G_{d 2 i 2}$ is well damped. At higher values, the dominant poles are defined by an undamped pole pair. In contrast, the zeros of the plant tend to value one and become dominant. On the one hand, these zeros are cancelled by the filter $H_{s i}$, which also influences the control plant from $G_{i 2 u c 2}$. On the other hand, the zeros of $G_{i 2 u c 2}$ are well damped and can be neglected. Because of the variations of the internal resistor, the design of the controller is difficult.

In contrast to this concept, an online system identification can estimate the model parameters. With the identification of the plant dynamic, the controller can re-design to ensure a)
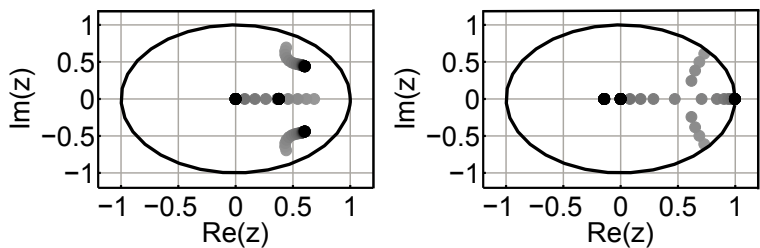

c)

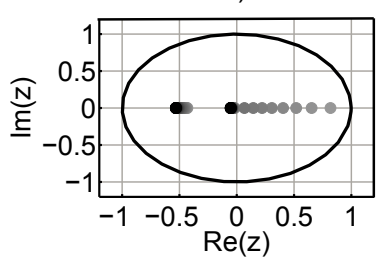

$3.0 \Omega$

$2.4 \Omega$

$1.8 \Omega$

$1.2 \Omega$

$0.6 \Omega$

$\operatorname{Re}(z)$

Fig. 7. Zero and Poles of the plants. a) shown the poles of $A$, b) shown the zeros of $B_{i}$ and c) shown the zeros of $B_{u}$ of different $r_{t e g}$ from $0 \ldots 3$ $\Omega$.

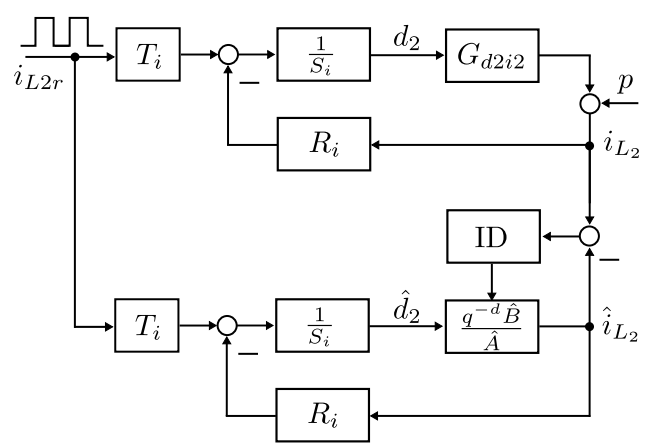

Fig. 8. Identification scheme of the current closed loop.

the desired closed loop dynamic and stability. The closed loop of the current control has a significant influence on the cascade control. To identify the parameters of the transfer function (10), the voltage loop is open and a square-wave signal is used as the current reference signal $i_{L 2 r}$. The feedback has a fixed controller and an adjustable model of the controlled system. The parameter estimation of the plant uses the adaption error

$$
\varepsilon=i_{L 2}-\hat{i}_{L 2}
$$

between the measured signal $i_{L 2}$ of the real system and the predicted $\hat{i}_{L 2}$ as a quality criterion. It should be noticed, that the tilde symbol is neglected in the following. The goal of the identification algorithm (ID) is to find the plant parameters, which minimize the predicted error.

The difficulty in a close loop identification is the correlation between the control signal $d_{2}$ and output signal $i_{L 2}$ [16]. The idea of the closed loop output error algorithm is, that the closed loop is considered to be an open loop, with knowing the controller filters. Hence, the regressors of the predictor error will be re-written [9]. 
The output of the plane (10) is given by

$$
\begin{aligned}
i_{L 2}(k+1)= & -A^{*} i_{L 2}(k)-B_{i}^{*} d_{2}(k-d) \\
& +A^{*} p(k+1) \\
= & \boldsymbol{\theta}^{T} \boldsymbol{\Phi}(k)+A^{*} p(k+1)
\end{aligned}
$$

where $d_{2}(k)$ is the plant input and $p(k)$ is the disturbance noise (see Fig. 8), and also

$$
\begin{aligned}
A= & 1+a_{1} q^{-1}+\ldots+a_{n_{a}} q^{-n_{a}} \\
= & 1+q^{-1} A^{*}\left(q^{-1}\right) \\
B= & b_{1} q^{-1}+\ldots+b_{n_{b}} q^{-n_{b}}=q^{-1} B^{*}\left(q^{-1}\right) \\
\boldsymbol{\theta}^{T}= & {\left[a_{1} \ldots a_{n_{a}} b_{1} \ldots b_{n_{b}}\right] } \\
\boldsymbol{\Phi}^{T}= & {\left[\begin{array}{lll}
-i_{L 2}(k) \ldots-i_{L 2}\left(k-n_{a}+1\right) \\
& d_{2}(k-d) \ldots d_{2}\left(k-d-n_{b}+1\right)
\end{array}\right] } \\
d_{2}(k)= & \frac{T_{i}}{S_{i}} i_{L 2 r}(k)-\frac{R_{i}}{S_{i}} i_{L 2}(k)
\end{aligned}
$$

The predictor of the closed loop is described by

$$
\hat{i}_{L 2}(k+1)=-\hat{A}^{*} \hat{i}_{L 2}(k)-\hat{B}_{i}^{*} \hat{d}_{2}(k-d)=\hat{\boldsymbol{\theta}}^{T} \hat{\boldsymbol{\Phi}}(k)
$$

where " " symbols the predictor values and parameters. With $(24$ - 30) can (20) be re-written to

$$
\varepsilon(k+1)=\frac{S_{i}}{P_{c l i}}(\boldsymbol{\theta}-\hat{\boldsymbol{\theta}})^{T} \boldsymbol{\Phi}(k)
$$

With the assumption that the disturbance $p(k)$ is white and not correlated with $\hat{\boldsymbol{\Phi}}$ the recursive least square (RLS) algorithm can be used

$$
\begin{aligned}
\hat{\boldsymbol{\theta}}(k+1) & =\hat{\boldsymbol{\theta}}(k)+\boldsymbol{L}(k) \varepsilon(k+1) \\
\boldsymbol{L}(k+1) & =\frac{1}{\lambda+\boldsymbol{\Phi}(k)^{T} \boldsymbol{P}(k) \boldsymbol{\Phi}(k)} \\
\boldsymbol{P}(k+1) & =\frac{1}{\lambda}\left[\boldsymbol{P}(k)-\frac{\boldsymbol{P}(k) \boldsymbol{\Phi}(k) \boldsymbol{\Phi}(k)^{T} \boldsymbol{P}(k)}{\lambda+\boldsymbol{\Phi}(k)^{T} \boldsymbol{P}(k) \boldsymbol{\Phi}(k)}\right]
\end{aligned}
$$

where $\boldsymbol{L}(k)$ is the adaption gain, $\boldsymbol{P}(k)$ the covariance matrix and $\lambda$ the forgetting factor [12]. The prediction can be validated with the correlation test about $N$ sample values [10]. The whiteness can be tested with the auto-correlation of the predictor error. The whiteness implies that the estimated parameters are unbiased and that they are uncorrelated with the disturbance. The confidence test is defined as

$$
\left|R N_{\varepsilon z}(i)\right| \leq \frac{\tau}{\sqrt{N}}, \quad z=\hat{i}_{L 2}, \varepsilon
$$

where $\tau$ implies the confidence interval.

\section{EXPERIMENT}

In Section III-B and IV the control structure and the algorithm for a self-tuning controller are presented. To verify the theoretical aspects, an experimental device is used to test the algorithm. The TEG is simulated with a constant voltage source and an internal resistor. The load is a $12.5 \mathrm{~V} 72 \mathrm{Ah}$ lead acid battery. The converter is controlled by a MicroAutoBox II 1401/1511/1512, with a sampling frequency of 10 $\mathrm{kHz}$. The converter is designed for a maximum current ripple of $15 \%$ and voltage ripple of $8 \%$. The parameters of the
TABLE I

PARAMETER OF BOOST-BUCK CONVERTER

\begin{tabular}{c|c||c|c||c|c}
\hline \hline$L_{1}$ & $45 \mu \mathrm{H}$ & $L_{2}$ & $22.4 \mu \mathrm{H}$ & $r_{L 1}$ & $43.2 \mathrm{~m} \Omega$ \\
$r_{L 2}$ & $17.7 \mathrm{~m} \Omega$ & $C_{1}$ & $20 \mu \mathrm{F}$ & $C_{2}$ & $88 \mu \mathrm{F}$ \\
$C_{3}$ & $30 \mu \mathrm{F}$ & $r_{C 1}$ & $1.2 \mathrm{~m} \Omega$ & $r_{C 2}$ & $17.1 \mathrm{~m} \Omega$ \\
$r_{C 3}$ & $0.8 \mathrm{~m} \Omega$ & $e_{b l}$ & $12.5 \mathrm{~V}$ & $r_{b l}$ & $100 \mathrm{~m} \Omega$ \\
\hline \multicolumn{4}{l}{$S_{1}, S_{2}, S_{3}, S_{4}$ Infineon IPP80N06S2L-07,$f_{s}=100 \mathrm{kHz}$} \\
\hline
\end{tabular}

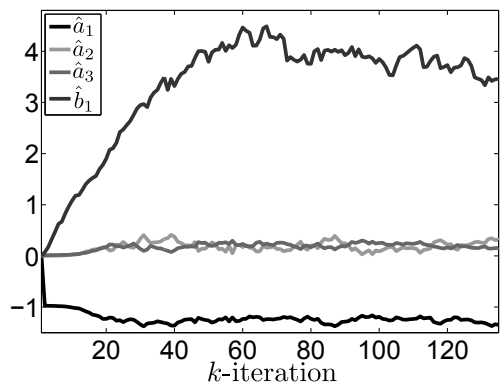

Fig. 9. The estimated parameters of plant for each iteration-step.

experimental boost-buck converter are presented in Table I. The equivalent series resistance are from the data sheets of the semiconductors.

The design of the pole placement from the current controller is based on the assumption, that the TEG has $u_{t e g}=20 \mathrm{~V}$ and an internal resistor $r_{\text {teg }}=0.3 \Omega$. The desired closed loop polynomial has two poles at 0.76 . For the experiment, the internal resistor is changed to $1.9 \Omega$.

For the self-tuning controller, the voltage feedback controller is open and a square-wave signal with a peak magnitude of $10 \%$ of the last reference signal $i_{L 2 r}$ from the voltage controller is used. The RLS is initialised with $n_{a}=3, n_{b}=1$ and $\lambda=0.998$. Fig. (9) shows the evolution of parameter estimation from the RLS. After 60 iterations, the parameters convert to a steady state. The algorithm starts at each 40th iterations-steps a model verification. The verification uses the correlation analysis (35). The confidence interval is $95 \%$, it follows that $|R N|<0.196$, by 100 samples with $\tau=1.96$

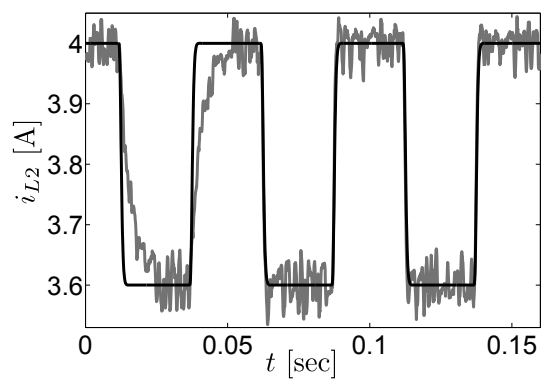

Fig. 10. Adaption of the control parameters, with $u_{t e g}=20 \mathrm{~V}, 1.8 \Omega$. Black line is the desired closed loop, gray line is the current closed loop. 
[10]. The confidence criterium between the error and the output prediction is $\left|R N_{\varepsilon i_{L 2}}\right|<0.065$ and the residual prediction error is $\left|R N_{\varepsilon \varepsilon}\right|<0.151$. This statistical test implies, that the identification is successful. Based on these values, the control parameters are re-designed (see Fig. 10). The proposed closed loop identification is successful for the presented experiment. For this application, the order of $n_{a}$ and $n_{b}$ is pre-specified. The problem is, that the zeros of the plant can be complex if the internal resistor of the TEG changes. In this case, the algorithm must be extended with order variation of $n_{b}$. Further, if the dominant zeros should be canceled with a filter from the current controller, the voltage closed loop must be re-designed as well. In this experiment, the designed poles are dominant against the zeros and canceling of the zeros is not necessary. The control design based on the SSA-models, with a maximum bandwidth of $f_{s} / 2$. The proposed application using lowpass filters, with a cut off frequency of $1 \mathrm{kHz}$. Thus, highfrequency influences are damped, so as comply with validity of the SSA model.

\section{CONCLUSIONS}

A digital control of a boost-buck converter for a TEG is presented. The converter can be modeled and analysed with the SSA. The control of the converter includes a MPPT, whose function it is to adapt the maximum power point of the TEG. Further, a cascade controller regulates the voltage between the two converters, and a subsidiary current controller regulates the charge current of the battery. The control design is based on a two-degrees-of-freedom controller, whose parameter are well defined for a TEG, with the assumption of a steady temperature. Variations of the temperature influence the voltage and especially the resistor of the TEG, which also affects the dynamic of the control plant. A method to compensate this influence, is a selftuning controller which is uses a closed loop identification to estimate the parameter of the plant. In the experiments a RLS algorithm was used to estimate the zeros and poles of the control plant. The online re-design of the control parameters could ensure the desired closed loop dynamic of the current controller.

\section{OUTLOOK}

The next step will be to implement a charging voltage control loop. Additional the self-tuning algorithm will be extended with an adaptive model order, which also detect control plants with not well damped zeros. Finally, the filter polynomial $H_{s i}$ of the voltage control must be updated, if the zeros of the current control plant should be canceled.

\section{APPENDIX}

$$
\begin{aligned}
& r_{d s 1}=r_{d s 2}=r_{d s 3}=r_{d s 4} \\
& \alpha_{1}=\frac{-r_{C 1} r_{L 1}-r_{t e g} r_{L 1}-r_{t e g} r_{C 1}}{L_{1}\left(r_{C 1}+r_{t e g}\right)} \\
& \alpha_{2}=\frac{r_{t e g}}{L_{1}\left(r_{C 1}+r_{t e g}\right)} \\
& \alpha_{3}=\frac{-r_{C 1}-r_{t e g}}{L_{1}\left(r_{C 1}+r_{t e g}\right)} \\
& \alpha_{4}=\frac{r_{C 1}}{L_{1}\left(r_{C 1}+r_{t e g}\right)} \\
& \alpha_{5}=\alpha_{6}=-\alpha_{8}=\frac{r_{C 1} r_{C 2}+r_{t e g} r_{C 2}}{L_{1}\left(r_{C 1}+r_{t e g}\right)} \\
& \alpha_{7}=\frac{r_{C 1}+r_{t e g}}{L_{1}\left(r_{C 1}+r_{t e g}\right)} \\
& \beta_{1}=\beta_{2} r_{t e g}=-\beta_{3}=-\frac{1}{C_{1}\left(r_{C 1}+r_{t e g}\right)} \\
& \delta_{1}=-\delta_{2}=-\delta_{3}=\frac{1}{C_{2}} \\
& \gamma_{2}=-r_{b l} \gamma_{1}=-\gamma_{3}=-\frac{1}{C_{3}\left(r_{C 3}+r_{b l}\right)} \\
& \epsilon_{1}=-\frac{r_{C 3} r_{L 2}+r_{b l} r_{L 2}+r_{b l} r_{C 3}}{L_{2}\left(r_{C 3}+r_{b l}\right)} \\
& \epsilon_{2}=\frac{r_{C 3}}{r_{b l}} \epsilon_{3}=-\frac{r_{b l}}{L_{2}\left(r_{C 3}+r_{b l}\right)} \\
& \epsilon_{4}=-\epsilon_{5}=\epsilon_{6}=\frac{r_{b l} r_{C 2}+r_{C 2} r_{C 3}}{L_{2}\left(r_{C 3}+r_{b l}\right)}
\end{aligned}
$$

\section{REFERENCES}

[1] J. H. Carstens and C. Gühmann. Simulative analysis and evaluation of $\mathrm{dc} / \mathrm{dc}$ converters for thermoelectric generators. In Simulation und Test für die Automobilelektronik IV, pages 145 -155. Expert Verlag, 2012.

[2] R. W. Erickson and D. Maksimovic. Fundamentals of Power Electronics. Springer, 2ed edition, 2001.

[3] T. Esram and P. L. Chapman. Comparison of photovoltaic array maximum power point tracking techniques. IEEE Transactions on Energy Conversion, 22(2):439-449, June 2007.

[4] H. J. Goldsmid and G. S. Nolas. A review of the new thermoelectric materials. In XX International Conference on Thermoelectrics, 2001. Proceedings ICT 2001, pages 1 -6. IEEE, 2001.

[5] R.-Y. Kim. Improved Renewable Energy Power System using a Generalized Control Structure for Two-Stage Power Converters. PhD thesis, Virginia Polytechnic Institute and State University, August 2009.

[6] J. Kitte, I. Friedrich, D. Jänsch, and A. Sommer. Opportunities of waste heat recovery or heat conditioning via thermoelectricity in passenger cars - a comparison of the system integration challenges. In Thermoelectrics Goes Automotive: Thermoelektrik, II, pages 249 -272. Expert Verlag, 2011.

[7] J. Kitte, R. Kühn, H.-F. Pernau, K. Littmann, and D. Jänsch. Dimensioning and evaluating a multi-channel thermoelectric generator using a customized simulation architecture. In Thermoelectrics Goes Automotive: Thermoelektrik, III, pages 207 -224. Expert Verlag, 2012.

[8] I. D. Landau and K. Alireza. Recursive algorithms for identification in close loop: A unified approach and evaluation. Automatica, 33(8):1499-1523, Mar. 1997.

[9] I. D. Landau and A. Karimi. Recursive algorithms for identification in closed loop: A unified approach and evaluation. Automatica, 33(8):1499-1523, Aug. 1997.

[10] I. D. Landau and G. Zito. Digital Control Systems: Design, Identification and Implementation. Springer, May 2007. 
[11] S. Lineykin and S. Ben-Yaakov. Modeling and analysis of thermoelectric modules. IEEE Transactions on Industry Applications, 43(2):505512, Apr. 2007.

[12] L. Ljung, editor. System identification (2nd ed.): theory for the user. Prentice Hall PTR, Upper Saddle River, NJ, USA, 1999.

[13] R. D. Middlebrook and S. Cuk. A general unified approach to modelling switching-converter power stages. In Power Electronics Specialists Conference, volume -1, pages 18 -34, 1976.

[14] D. M. Rowe, editor. Modules, Systems and Applications in Thermoelectrics. CRC Press, 2012.

[15] A. Shakouri and S. Li. Thermoelectric power factor for electrically conductive polymers. In Eighteenth International Conference on Thermoelectrics, 1999, pages 402 -406. IEEE, 1999.

[16] R. J. P. Van Den Hof, Paul M. J.and Schrama. Identification and control - closed-loop issues. IEEE Transactions on Industry Applications, 31(12):1751-1770, 1995.

[17] W. Xiao and W. Dunford. A modified adaptive hill climbing MPPT method for photovoltaic power systems. In Power Electronics Specialists Conference, 35th Annual, volume 3, pages 1957-1963. IEEE, 2004. 